\title{
CRP, D-dimer, fibrinogen and ESR as predictive markers of response to standard doses of levocetirizine in patients with chronic spontaneous urticaria
}

Department of Dermatology and Venereology, I.M. Sechenov First Moscow State Medical University, Moscow, Russia

KEY WORDS

chronic spontaneous urticaria;

levocetirizine; ESR; CRP; D-dimer;

fibrinogen

\author{
Corresponding author \\ Pavel Kolkhir \\ I.M. Sechenov First Moscow State Medical \\ University \\ Department of Dermatology and Venereology \\ 4/2, B. Pirogovskaya St., Moscow, 119991 \\ Phone: +8 (495) 6091400 \\ E-mail: pavel.kolkhir@yandex.ru
}

Doi

10.23822/EurAnnACI.1764-1489.05

\begin{abstract}
Summary
According to current guidelines, non-sedative H1-antihistamines (nsAH) are the first-line therapy of chronic spontaneous urticaria (CSU). But even up-dosed antihistamines (to four times the standard dose) produce symptom resolution in less than 50\% of patients. Biomarkers that can predict the response to nsAH are still unknown. We carried out a prospective study and used discriminant analysis to evaluate the combination of D-dimer, fibrinogen, $C$-reactive protein and ESR values for predicting the outcome of treatment with levocetirizine in 84 CSU patients. We found that elevation of these parameters is associated with more active disease, low quality of life and lack of response to standard doses of levocetirizine. Thus, evaluation of these markers may be considered useful before starting treatment with ns AH. The mechanisms behind the increase in these parameters in CSU patients need to be elucidated in further studies.
\end{abstract}

\section{To the Editor}

Chronic spontaneous urticaria (CSU) is defined as recurrence of wheals, angioedema, or both for $>6$ weeks due to known or unknown causes. According to current guidelines, non-sedative $\mathrm{H} 1$-antihistamines (nsAH) are the first-line therapy of CSU (1). But even up-dosing to four times the standard dose can provide symptom control in only less than $50 \%$ of CSU patients (2). Identification of biomarkers for predicting the response to nsAH is necessary for developing an individualized treatment. Total IgE, D-dimer, fibrinogen, ESR and C-reactive protein (CRP) have been previously investigated as potential biomarkers of CSU activity and/or response to treatment (3-5). Herein, we for the first time evaluated their combination for predicting the outcome of treatment with levocetirizine in CSU patients.
This prospective study included 84 CSU patients seen from 2012 to 2015 at our Department. Patients with inducible urticaria, urticarial vasculitis and those, receiving anticoagulants, steroids or immunosuppressive agents were excluded. The patients had no history of allergies, thrombosis and chronic infections.

On day 0 , all patients stopped taking antihistamines for 5 days. They filled out the Russian versions of Chronic Urticaria Quality of Life Questionnaire (CU-Q2oL) and Urticaria Activity Score (UAS) on daily basis during this period. On day 5 , serum levels of total IgE, D-dimer and fibrinogen were measured using ELISA, CRP - high-sensitive nephelometric method. ESR was determined by Westergren method. Autologous serum skin test (ASST) was performed to assess autoreactivity / autoimmunity. Levocetirizine was then administrated at the dose of $5 \mathrm{mg} /$ day 
for 7 days. The efficacy of therapy was evaluated based on CUQ2oL and UAS scores.

Statistical analysis was performed using SPSS v.22.0 (IBM, New York, USA). Mann-Whitney U-test, Pearson correlation coefficient, chi-square criterion $\chi^{2}$ and Cramer's $V$ were employed for comparison between the parameters. Discriminant analysis was carried out to reveal predictive markers of response to levocetirizine. We were interested in the relationship between a group of independent variables (ESR, D-dimer, fibrinogen, CRP, total IgE, UAS and CU-Q2oL) and one categorical variable (efficacy of levocetirizine). ESR, D-dimer, fibrinogen, CRP, total IgE values were transformed to normal distribution. A p-value $\leq 0.05$ was considered statistically significant.

Of 84 CSU patients (aged 16-77 years), 25 (29.8\%) were males and $59(70.2 \%)$ were females. ASST positive patients had significantly more active / severe disease (mean rank: 42.84 vs
31.13, $\mathrm{U}=895, \mathrm{p}=0.019$ ), higher serum levels of CRP (mean rank: 43.02 vs $31.94, \mathrm{U}=901, \mathrm{p}=0.031$ ) and $\mathrm{D}$-dimer (mean rank: 42.97 vs $30.95, \mathrm{U}=900.5, \mathrm{p}=0.019$ ) than those with negative ASST.

All patients were divided into two groups based on response to levocetirizine for discriminant analysis: responders $(n=42$, $50 \%)$ and non-responders $(n=42,50 \%)$. Levocetirizine was more often effective in patients with mild CSU compared to those with severe disease (66.7 vs $22.9 \%, \chi^{2}=3.696, \mathrm{p}=0.05$, $\mathrm{V}=0.228)$.

Of 42 responders, $7.1 \%, 9.5 \%, 12 \%, 31 \%$ and $47.6 \%$, had high serum levels or values of D-dimer, fibrinogen, ESR, CRP and total $\mathrm{IgE}$, respectively. Of 42 non-responders, 54.8\%, $26.2 \%, 35.7 \%, 54.8 \%$ and $23.8 \%$ demonstrated elevated D-dimer, fibrinogen, ESR, CRP and total IgE, respectively.

The most important results of discriminant analysis are summarized in tables $\mathbf{1}$ and $\mathbf{2}$. Mean values of parameters in table $\mathbf{1}$

Table 1 - Discriminant analysis. Distribution of observations into two groups based on efficacy of levocetirizine.

\begin{tabular}{|c|c|c|c|c|c|}
\hline \multirow{2}{*}{ Efficacy of levocetirizine } & \multirow{2}{*}{ Parameters } & \multirow{2}{*}{ Mean } & \multirow{2}{*}{$\begin{array}{l}\text { Standard } \\
\text { deviation }\end{array}$} & \multicolumn{2}{|c|}{ Valid N (listwise) } \\
\hline & & & & Unweighted & Weighted \\
\hline \multirow{7}{*}{ Effective } & ESR & 0.7151 & 0.39204 & 42 & 42.000 \\
\hline & D-dimer & 2.1248 & 0.38680 & 42 & 42.000 \\
\hline & CRP & 0.2859 & 0.67520 & 42 & 42.000 \\
\hline & UAS & 3.3810 & 1.48081 & 42 & 42.000 \\
\hline & CU-Q2oL & 63.8143 & 13.22093 & 42 & 42.000 \\
\hline & Fibrinogen & 0.4647 & 0.09294 & 42 & 42.000 \\
\hline & Total IgE & 1.9068 & 0.75460 & 42 & 42.000 \\
\hline \multirow{7}{*}{ Not effective } & ESR & 1.0797 & 0.35109 & 42 & 42.000 \\
\hline & D-dimer & 2.6728 & 0.47994 & 42 & 42.000 \\
\hline & CRP & 0.7965 & 0.58296 & 42 & 42.000 \\
\hline & UAS & 4.3095 & 1.48961 & 42 & 42.000 \\
\hline & CU-Q2oL & 49.8214 & 15.44157 & 42 & 42.000 \\
\hline & Fibrinogen & 0.5384 & 0.13369 & 42 & 42.000 \\
\hline & Total IgE & 1.5266 & 0.78568 & 42 & 42.000 \\
\hline \multirow{7}{*}{ Total } & ESR & 0.8974 & 0.41285 & 84 & 84.000 \\
\hline & D-dimer & 2.3988 & 0.51347 & 84 & 84.000 \\
\hline & CRP & 0.5412 & 0.67751 & 84 & 84.000 \\
\hline & UAS & 3.8452 & 1.54837 & 84 & 84.000 \\
\hline & CU-Q2oL & 56.8179 & 15.92696 & 84 & 84.000 \\
\hline & Fibrinogen & 0.5016 & 0.12029 & 84 & 84.000 \\
\hline & Total IgE & 1.7167 & 0.78918 & 84 & 84.000 \\
\hline
\end{tabular}


Table 2 - Discriminant analysis. Within-groups correlation matrix.

\begin{tabular}{lccccccc}
\hline Parameters & ESR & D-dimer & CRP & UAS & CU-Q2oL & Fibrinogen & Total IgE \\
\hline ESR & 1.000 & 0.289 & 0.410 & 0.161 & -0.140 & 0.431 & -0.034 \\
\hline D-dimer & 0.289 & 1.000 & 0.440 & 0.200 & -0.318 & 0.479 & -0.153 \\
\hline CRP & 0.410 & 0.440 & 1.000 & 0.139 & -0.280 & 0.357 & -0.132 \\
\hline UAS & 0.161 & 0.200 & 0.139 & 1.000 & -0.512 & 0.089 & -0.300 \\
\hline CU-Q2oL & -0.140 & -0.318 & -0.280 & -0.512 & 1.000 & -0.206 & 0.265 \\
\hline Fibrinogen & 0.431 & 0.479 & 0.357 & 0.089 & -0.206 & 1.000 & 0.120 \\
\hline Total IgE & -0.034 & -0.153 & -0.132 & -0.300 & 0.265 & 0.120 & 1.000 \\
\hline
\end{tabular}

show that responders have higher levels of total $\operatorname{IgE}$ and lower levels / values of D-dimer, fibrinogen, CRP and ESR, higher CU-Q2oL and lower UAS scores compared to non-responders. The results of univariate ANOVA's, carried out for each independent variable showed that ESR $(p<0.001)$, D-dimer $(p<$ $0.001)$, CRP $(p<0.001)$, fibrinogen $(p=0.004)$, UAS $(p=$ $0.005)$, CU-Q2oL $(\mathrm{p}<0.001)$ and total $\operatorname{IgE}(\mathrm{p}=0.026)$ significantly differ for responders and non-responders.

Within-groups correlation matrix shows correlations between predictors (table 2). There is a positive correlation between D-dimer, fibrinogen, CRP and ESR. As already mentioned, high levels of these are associated with lack of response to levocetirizine (table 1). There is also a negative correlation between values of D-dimer and CU-Q2oL, CRP and CU-Q2oL, total $\mathrm{IgE}$ and UAS, CU-Q2oL and UAS. Only total $\mathrm{IgE}$ correlates positively with CU-Q2oL.

Wilks' lambda is $0.620,\left(\chi^{2}=37.471, \mathrm{p}<0.001\right)$ indicating a good discriminatory ability of the discriminant function to distribute cases into groups.

The summary of number of subjects classified correctly and incorrectly for responders are $33(78.6 \%)$ and $9(21.4 \%)$, respectively. The summary of number of subjects classified correctly and incorrectly for non-responders are $34(81 \%)$ and 8 (19.0\%), respectively. The discriminant function worked equally well for each group of dependent variable. Overall, $79.8 \%$ of cases are correctly classified.

To the best of our knowledge, we were the first to assess the set of biomarkers in the same group of CSU patients for predicting response to nsAH. The role of $\mathrm{D}$-dimer and activation of coagulation in CSU pathogenesis is widely discussed (3-5). Patients with high levels of D-dimer, fibrinogen and/or CRP are shown to have severe $\operatorname{CSU}(3,5,6)$ that is in the line with the results of our study.

We reported elevation of total IgE serum levels in responders with mild CSU that is contradictory to the findings by Kessel and co-workers where total $\mathrm{IgE}$ was suggested as a marker of severe CSU (7). Thus, the role of total IgE in CSU should be better characterized in further research.

IgG-anti-IgE/FceRI autoantibodies play a role in a subpopulation of CSU patients (8). We observed that positive ASST is associated with more severe disease, higher serum levels of CRP and D-dimer. Obtained results suggest that positive ASST is more often seen in non-responders.

Thus, we found that the elevation of CRP, ESR, D-dimer and fibrinogen is associated with more active disease, low quality of life and lack of response to standard doses of levocetirizine. Evaluation of these markers may be considered before starting treatment with nsAH. Further research into the mechanisms behind elevation of these parameters in antihistamine-resistant $\mathrm{CSU}$ is required.

\section{Learning points}

- CRP, ESR, D-dimer and fibrinogen seem to be useful markers for prediction of response to levocetirizine in CSU patients.

- Elevation of these markers is associated with more active disease, low quality of life, and a lack of response to standard doses of levocetirizine.

- Evaluation of these parameters may be considered before starting the treatment with non-sedative antihistamines, and provides additional justification for administration of high doses of antihistamines. 


\section{References}

1. Zuberbier T, Aberer W, Asero R, Bindslev-Jensen C, Brzoza Z, Canonica GW, et al. The EAACI/GA(2) LEN/EDF/WAO Guideline for the definition, classification, diagnosis, and management of urticaria: the 2013 revision and update. Allergy. 2014;69(7):868-87.

2. Humphreys F, Hunter JA. The characteristics of urticaria in 390 patients. Br J Dermatol. 1998;138(4):635-8.

3. Asero R. D-dimer: a biomarker for antihistamine-resistant chronic urticaria. J Allergy Clin Immunol. 2013;132(4):983-6.

4. Kolkhir P, Andre F, Church MK, Maurer M, Metz M. Potential blood biomarkers in chronic spontaneous urticaria. Clin Exp Allergy. 2017;47(1):19-36.
5. Takeda T, Sakurai Y, Takahagi S, Kato J, Yoshida K, Yoshioka A, et al. Increase of coagulation potential in chronic spontaneous urticaria. Allergy. 2011;66(3):428-33.

6. Ucmak D, Akkurt M, Toprak G, Yesilova Y, Turan E, Yildiz I. Determination of dermatology life quality index, and serum C-reactive protein and plasma interleukin- 6 levels in patients with chronic urticaria. Postepy Dermatol Allergol. 2013;30(3):146-51.

7. Kessel A, Helou W, Bamberger E, Sabo E, Nusem D, Panassof J, et al. Elevated serum total IgE - a potential marker for severe chronic urticaria. Int Arch Allergy Immunol. 2010;153(3):288-93.

8. Kolkhir P, Church MK, Weller K, Metz M, Schmetzer O, Maurer M. Autoimmune chronic spontaneous urticaria: what we know and what we don't know. J Allergy Clin Immunol. 2017;139(6):1772-1781. 